\title{
Mortalidad por hipertermia en Bizkaia durante la ola de calor del verano de 2003: experiencia forense.
}

\author{
Heat-related mortality in Bizkaia during the Summer \\ 2003 heat wave. Forensic experience.
}

\author{
B. Morentin'1 , B. Biritxinaga ${ }^{2}$ y T. Peciña ${ }^{3}$
}

\section{RESUMEN}

Durante las olas de calor la mortalidad puede incrementar marcadamente, a veces hasta alcanzar proporciones epidémicas, como ha ocurrido en Francia este verano. Según las estadísticas oficiales provisionales en el País Vasco hubo 3 casos de muerte por golpe de calor.

Describimos 2 casos de muerte por golpe de calor (hipertermia) diagnosticas en el Departamento de Patología Forense de Bizkaia ocurridas durante la ola de calor que azotó a Europa el pasado verano. Ninguna de ellas fue incluida en las estadísticas oficiales provisionales.

Se trataba de 2 varones adultos con factores de riesgo: alcoholismo e intoxicación alcohólica en uno y cardiopatía y posible sobreejercicio en el otro. Se describen los resultados de la autopsia que mostraron quemaduras térmicas de $2^{\circ}$ grado y hallazgos histopatológicos inespecíficos. El estudio bioquímico de humor vítreo mostró un patrón de deshidratación en uno de ellos. La temperatura rectal fue de $41^{\circ} \mathrm{C}$ en uno $y$ de $43^{\circ} \mathrm{C}$ en el otro.

Este trabajo demuestra la importancia de la toma de la temperatura rectal y ambiente en situaciones de posible muerte por golpe de calor. El diagnóstico final requiere una valoración integrada de todos los datos circunstanciales, médicos, patológicos y de laboratorio. También subraya la conveniencia de un flujo rápido de la información desde el sistema forense al sistema sanitario encargado de la epidemiología y prevención del golpe de calor en situaciones de alarma.

Palabras clave: Hipertemia, Golpe de calor, Patología forense, Mortalidad.

\section{ABSTRACT}

During severe heat waves, like that experienced in the summer of 2003 in southern Europe, mortality can increase sharply, sometimes even acquiring epidemic proportions. According to the provisional official reports in the Basque Country there were 3 deaths by heatstroke.

In this article we describe the experience of the Forensic Pathologhy Departament of Bizkaia during the heat wave last summer- Autopsy reports were reviewed. Two deaths were due to heat stroke (fatal hyperthermia), but neither of them had been included in the provisional official reports.

Both cases were adult males with known risk factors: in the first one alcoholic liver disease and acute alcohol intoxication; and in the second one hypertensive cardiopathy and possible overexertion. In both cases there were external thermic injuries and rectal temperature was $>41^{\circ} \mathrm{C}$. The histological findings were nonspecific. In the first case vitreous humor electrolyte analysis suggested dehydration.

This article shows the importance of information about rectal and ambient temperature in the diagnosis of heat stroke, because autopsy findings are nonspecific. The definitive diagnosis requires a valoration of all the information: medical, pathological and laboratory data, as well as facts of the circumstances of the death. This, also, proves the convenience of fluid information from forensic systems to health departments, in charge of the epidemiolgy and prevention of heat stroke in alarm situations.

Key words: Heat-related deaths, Hyperthermia, Forensic Pathology, Mortality.

Correspondencia: Dr. Benito Morentin. Servicio de Patología Forense de Bizkaia. Instituto Vasco de Medicina Legal. C/ Barroeta Aldamar, 10. Planta 1. 48001. Bilbao (Bizkaia).Tfno: 9440167 31. Correo electrónico: MORENTIN.B@AJU.EJ-GV.ES

\footnotetext{
1 Médico forense. Doctor en Medicina. Servicio de Patología Forense de Bizkaia. Instituto Vasco de Medicina Legal.

2 Médico especialista en Anatomía Patológica. Servicio de Patología Forense de Bizkaia. Instituto Vasco de Medicina Legal.

3 Médico Forense. Servicio de Clínica Médico Forense. Instituto Vasco de Medicina Legal.
} 


\section{INTRODUCCIÓN:}

El verano que acaba de finalizar pasará a la historia meteorológica vasca como el más caluroso de las últimas décadas. En Bizkaia en la estación de Loiu el mercurio se quedó solo a una décima del record de $42^{\circ} \mathrm{C}$ de julio de 1947. Durante la primera quincena de agosto varios días tuvieron máximas superiores a los $35^{\circ} \mathrm{C}$ (según datos oficiales de temperaturas medidas a la sombra). Al sol los termómetros alcanzaron los $50^{\circ} \mathrm{C}[\mathrm{I}]$. Las consecuencias de esta ola de calor se han dejado notar en distintos ámbitos, entre otros el sanitario. En España, las cifras provisionales del Ministerio de Sanidad y Consumo a fecha 3 de octubre de 2003 eran de que a consecuencia de la ola de calor fallecieron 14I personas: 59 de ellas por golpe de calor y 82 por agravamiento de la patología previa [2]. En la Comunidad Autónoma del País Vasco se registraron 3 muertes, todas ellas ocurridas en hospitales [2]. La situación fue más grave en otros países de Europa, sobre todo en Francia, donde se dieron cifras de verdadera epidemia.

Las cifras ofrecidas hasta el momento, al menos en Bizkaia, no han tenido en consideración la mortalidad investigada por los servicios forenses [3]. Sin embargo, una proporción no despreciable de las muertes por hipertermia son investigadas por la medicina forense, sobre todo cuando la muerte ocurre extrahospitalariamente y afecta a personas no ancianas y a personas sin enfermedad previa conocida potencialmente letal [3].

El objetivo del presenta trabajo era valorar la magnitud del problema de la mortalidad por la ola de calor del verano de 2003 en el contexto forense y valorar las circunstancias, factores de riesgo y hallazgos patológicos de este tipo de muertes.

\section{MATERIAL Y MÉTODO:}

Los archivos del Servicio de Patología Forense de Bizkaia desde mayo a septiembre de 2003 fueron revisados. Se seleccionaron los casos en los que constaba como causa fundamental de muerte hipertermia / golpe de calor por exposición a altas temperaturas ambientales. Los datos relativos a los antecedentes patológicos, circunstancias en torno al fallecimiento, hallazgos de autopsia y estudios histopatológicos, químico-toxicológicos y bioquímicos fueron revisados. Los rangos de normalidad expresados en el estudio bioquímico son valores aplicables a humor vítreo en sujetos fallecidos [4].

\section{RESULTADOS:}

En el periodo analizado se identificaron 2 casos de muerte por golpe de calor. En ambos se practicó una autopsia completa con estudio histopatológico, toxicológico y bioquímico, estos 2 últimos en colaboración con el Instituto de Toxicología de Madrid.

\section{CASO No1.}

Varón de 56 años con antecedentes de alcoholismo crónico, neuropatía periférica y distocia social (vagabundo). Fue encontrado en una plaza a las 15 horas en situación de parada cardiorrespiratoria por los equipos de emergencias. Según testigos presénciales llevaba varias horas bajo exposición directa al sol. La temperatura ambiental era de $44^{\circ} \mathrm{C}$. La temperatura rectal a las 17 horas (a su ingreso en el Servicio de Patología) era de $41^{\circ} \mathrm{C}$. En el examen externo se apreciaron extensas lesiones tipo quemadura de $2^{\circ}$ grado con despegamiento epidérmico y formación de ampollas en tronco y en extremidades (figura $n^{\circ}$ I). La autopsia excluyó otras causas de muerte. Los hallazgos principales fueron esteatosis hepática masiva (figura $n^{\circ} 2$ ) y enfermedad ateromatosa coronaria de grado moderado. El examen histológico de las lesiones de la piel mostró áreas 
con amplia separación dermo-epidérmica con formación de ampollas subepidérmicas, desorganización de la arquitectura celular normal de la epidermis, elongación vertical de los queratinocitos y compactación de la dermis; en otras áreas existía extenso desprendimiento de la epidermis (hallazgos característicos de las injurias por quemadura térmica de $2^{\circ}$ grado).

La concentración de alcohol etílico fue de $2.2 \mathrm{~g} / \mathrm{L}$ en sangre, de $2.9 \mathrm{~g} / \mathrm{L}$ en humor vítreo y de $3.5 \mathrm{~g} / \mathrm{L}$ en orina. Los resultados del estudio bioquímico fueron los siguientes: cloruros 153 $\mathrm{mEq} / \mathrm{L}$ (rango de normalidad de I05- I32), urea I, I 2 g/L (rango de normalidad de <0,6) y creatinina I,6 mg/l $00 \mathrm{ml}$ (rango de normalidad de 0,6-1,36).

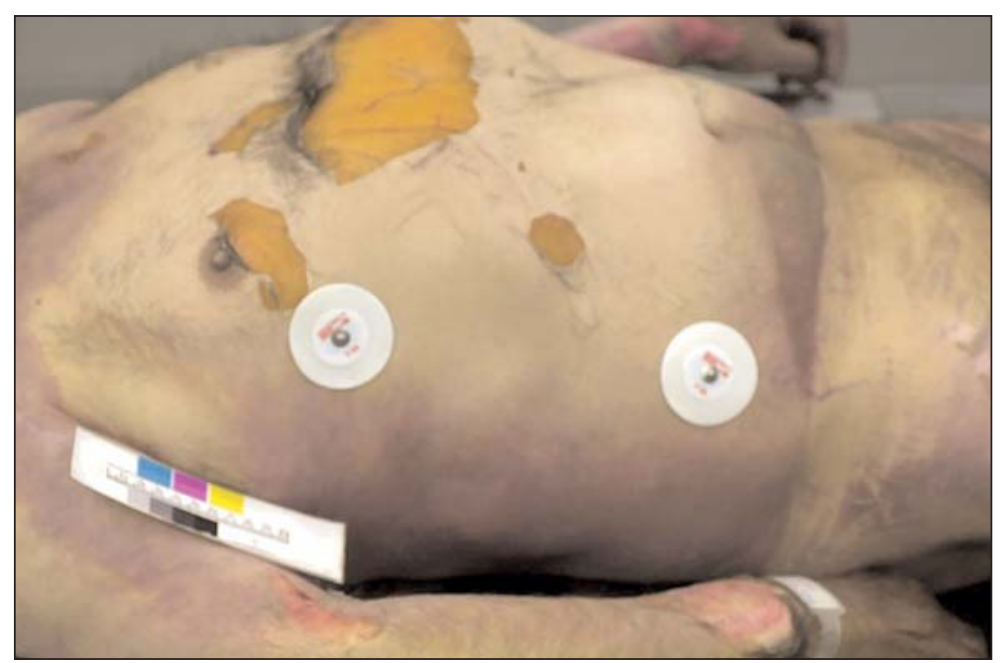

Figura 1. Lesiones por quemadura térmica en superficie de tronco y extremidades superiores (caso $\left.n^{\circ} 1\right)$.

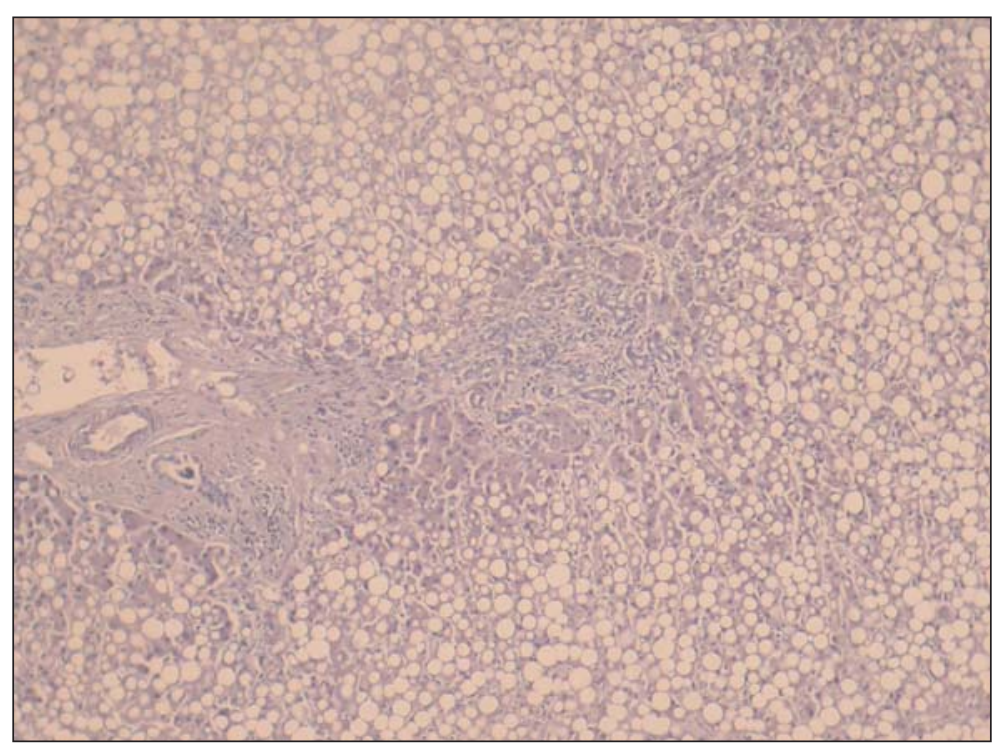

Figura 2. Esteatosis hepática masiva (caso $n^{\circ}$ 1) (Hematoxilina-eosina). 


\section{CASO N 2.}

Varón de 78 años. Antecedentes médico quirúrgicos de laringectomía y cardiopatía. En tratamiento farmacológico con nifedipino, ácido acetil salićlico, acenocumarol, diltiazem y pravastatina. Los días previos no había manifestado ningún tipo de sintomatología prodrómica. Fue encontrado a las 19 horas en parada cardiorrespiratoria en una huerta donde había estado trabajando. La temperatura ambiente era de $43,5^{\circ} \mathrm{C}$. La temperatura rectal a las 20 horas (hora de intervención médico forense) fue de $43^{\circ} \mathrm{C}$. Se apreciaron lesiones en región esternal y en extremidades inferiores de tipo ampolloso con despegamiento de la epidermis. Los hallazgos de autopsia fueron edema cerebral (peso del encéfalo de 1625 gramos), hipertrofia cardiaca patológica de origen hipertensivo, enfermedad ateromatosa coronaria de grado leve, microhemorragias pulmonares (figura $n^{\circ} 3$ ) y congestión vascular generalizada. El estudio histológico de las lesiones en superficie externa mostró hallazgos similares a los del caso $n^{\circ}$ I (figura 4). El estudio bioquímico de humor vítreo mostró unos valores dentro de la normalidad. El estudio químico toxicológico detectó solo el fármaco diltiazem en concentraciones terapéuticas.

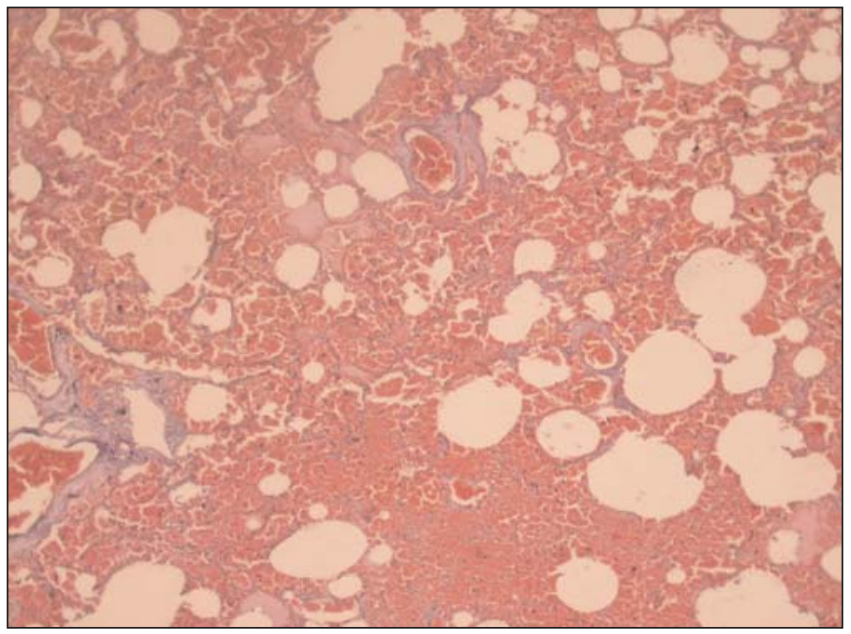

Figura 3. Microhemorragias pulmonares (caso $n^{\circ}$ 2) (Hematoxilina-eosina).

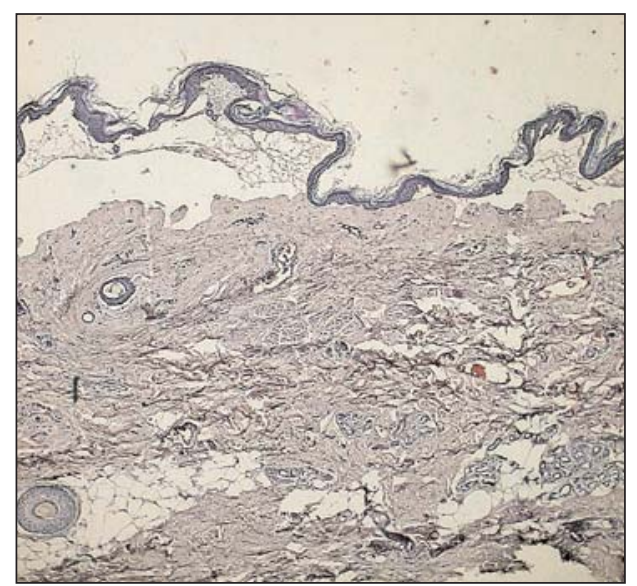

Figura 4. Examen histológico de la piel obtenida a nivel de las lesiones, mostrando amplia separación dermoepidérmica con formación de ampollas subepidérmicas (Hematoxilina-eosina). 
En ambos casos se concluyó que la causa de la muerte era una hipertermia (golpe de calor) por exposición a temperatura ambiental muy alta.

\section{DISCUSIÓN:}

El golpe de calor es una situación en el que una persona sometida a altas temperaturas ambientales experimenta un fracaso, potencialmente letal, del sistema de termorregulación corporal. Durante las olas de calor la mortalidad puede incrementar marcadamente, a veces hasta alcanzar proporciones epidémicas, como ha ocurrido en Francia este verano.

El sistema forense tiene el deber de diagnosticar adecuadamente las muertes por golpe de calor cuando estas ocurren en su ámbito de investigación. Ello ayudaría a ofrecer datos epidemiológicos fiables sobre mortalidad y contribuir así a instaurar medidas preventivas ante situaciones de alarma [5,6], como la vivida este último verano en algunas partes de Europa. Sin embargo, las cifras oficiales manejadas hasta ahora no han tenido en cuenta (al menos según la experiencia de Bizkaia) la mortalidad forense.

Entre las personas en riesgo de muerte por golpe de calor están los ancianos (sobre todo aquellos que viven aislados socialmente); las personas con enfermedades previas como insuficiencia cardiaca congestiva, enfermedades cerebrovasculares y alcoholismo; los pacientes sometidos a determinados tratamientos farmacológicos; $y$ las personas intoxicadas con drogas depresoras del sistema nerviosos central y con alcohol [5-8]. El sobreejercicio y la vestimenta inapropiada pueden contribuir a los efectos severos del calor [8]. Los 2 casos presentados en este trabajo presentaban factores de riesgo: alcoholismo crónico con esteatosis masiva e intoxicación aguda por alcohol etílico en uno y cardiopatía hipertensiva y posible sobreejercicio en el otro.

Los pacientes de golpe de calor que llegan vivos a un servicio de urgencias presentan un cuadro clínico característico con hipertermia severa, alteración del nivel de conciencia y anhidrosis [7]. Para efectuar el diagnóstico es imprescindible la exclusión de otras causas de hipertermia. La muerte de estos pacientes generalmente no presentan problemas de diagnósticos o de certificación.

El diagnóstico de golpe de calor en personas encontradas muertas es más difícil, y es aún más complicado cuando el cuerpo se encuentra en avanzado estado de putrefacción. Los hallazgos de autopsia son mínimos e inespecíficos, siendo difíciles de interpretar. Cuando sobreviven menos de 12 horas los hallazgos son pequeños o ausentes: puede observarse hemorragias petequiales sobre la superficie de los pulmones, corazón y otras serosas. Posteriormente puede aparecer edema pulmonar y edema cerebral. En una serie se ha descrito la existencia en varios casos de hemorragia pulmonar severa o de hemorragia subendocárdica en ventrículo izquierdo. Cuando la supervivencia es más prolongada se puede apreciar necrosis centrolobulillar hepática, necrosis tubular aguda renal, degeneración neuronal, pancreatitis aguda y en caso de coagulación intravascular diseminada trombos de fibrina en pequeños vasos sanguíneos. Otros hallazgos descritos han sido desprendimiento epidérmico y lesiones por quemadura térmica $[5,8-10]$. Nuestros casos mostraron hallazgos escasos, probablemente por su corta supervivencia. En ambos se objetivaron lesiones térmicas por quemadura y en uno de ellos hemorragia pulmonar y edema cerebral.

El análisis bioquímico en el humor vítreo puede mostrar parámetros de deshidratación [8], si bien los resultados son poco interesantes en cadáveres con periodo postmortem prolongado. En el caso $n^{\circ} \mid$ el aumento de los cloruros, de la urea y la creatinina es compatible con el patrón bioquímico de deshidratación postmortem definido por varios autores $[4, \mathrm{I} I]$. Patrones similares de alteraciones bioquímicas ha sido observado en pacientes hospitalarios. En una serie clínica de 78 casos de golpe de calor la elevación en la concentración de urea fue observada en el $86 \%$ e hipernatremia en el $46 \%$ [7]. 
La sospecha inicial de golpe de calor debería ser tenida en cuenta en cualquier persona expuesta a altas temperaturas. El diagnóstico final se debe basar en una valoración global de todos los datos: a) historia de exposición a altas temperaturas ambientales ( $\left.>37.8^{\circ} \mathrm{C}\right)$; b) exclusión de otras causas de muerte; c) exclusión de otras causas de hipertermia; d) análisis de los hallazgos macroscópicos, histopatológicos, toxicológicos y bioquímicos de la autopsia; e) valoración de la sintomatología prodrómica, de los antecedentes patológicos y de los factores de riesgo de la hipertermia; y f) en la medición precoz de la temperatura rectal en cadáveres recientes. En sujetos que inicialmente son encontrados con vida y posteriormente mueren, el diagnóstico definitivo de muerte por golpe de calor se alcanza cuando la temperatura corporal antemortem (en el momento del colapso) es superior a los $40.6^{\circ} \mathrm{C}[5]$.

La falta de conocimiento del golpe calor por parte del médico forense puede contribuir a que este cuadro pase desapercibido y sea mal diagnosticado. Uno de los datos principales es la toma precoz de la temperatura rectal. Por desgracia, solo en raras ocasiones esta medición es recogida en las personas encontradas cadáver, con lo que el diagnóstico se debe basar casi exclusivamente en datos circunstanciales. En una época en la que la medicina clínica, y también cada vez más la medicina forense, está acostumbrada a instrumentos diagnósticos muy sofisticados y de elevado coste económico, parecemos olvidar que procedimientos muy simples y baratos pueden ser de gran utilidad. El termómetro es uno de ellos, y su uso debería ser obligatorio en toda situación en la que se pueda sospechar un golpe de calor a la vista de los datos circunstanciales: días de altas temperaturas ambientales, niños dejados cerrados en un coche aparcado al sol, recién nacidos encontrados muertos en la cama con mucha ropa, etc.

\section{AGRADECIMIENTOS:}

Los autores desean agradecer las constructivas críticas de Gloria Vallejo y de Javier Capilla (Servicio de Biología del Instituto Nacional de Toxicología y Ciencias Forenses de Madrid) sobre la interpretación de los datos bioquímicos de humor vítreo; así como a la Técnica Especialista en Anatomía Patológica, Aída Larrañaga, por la asistencia técnica y a Ricardo Ballesteros por su ayuda en la traducción del resumen al inglés.

\section{BIBLIOGRAFÍA:}

I.- Diario GARA. 21 de septiembre de 2003. Páginas 2,3.

2.- El Médico Interactivo [diario electrónico] 2003,. 03 de octubre de 2003, n 1054. Disponible en: titulares@medynet.com. 3.- Morentin B, Alcaraz A. Mortalidad por golpe de calor en medicina forense. Implicaciones epidemiológicas. Med Clin (Barc) 2003; 121:796-7. 4.- Coe JI. Postmortem chemistry update. Emphasis on forensic application. Am J Forensic Med Pathol 1993; 14: 91-II7.

5.- Lifschultz BD, Donoghue ER. Forensic pathology of heat- and coldrelated injuries. Clinics in Laboratory Medicine 1998; 18: 18: 77-89.

6.- Wolfe MI, Kaiser R, Naughton MP, Mirabelli MC, Yoon SS, Hanzlick $\mathrm{R}$, Henderson AK. Heat-related mortality in selected United States Cities, summer 1999. Am J Forensic Med Pathol 2001; 22: 352-7.

7.- Jiménez-Mejias ME, Montano Diaz M, Villalonga J, Bollain Tienda E, Lopez Pardo F, Pineda JA, et al. Golpe de calor clásico en España. Análisis de una serie de 78 casos. Med Clin (Barc) 1990; 94: 481-6.

8.- Green H, Gilbert J, James R, Byard RW. An analysis of factors contributing to a series of deaths caused by exposure to high environmental temperatures. Am J Forensic Med Pathol 200l; 22: 196-9.

9.- Donoghue ER, Graham MA, Jentzen JM, Lifschultz BD, Luke JL, Mirchandani HG. Criteria for the diagnosis of heat-related deaths: National association of medical examiners. Position Paper. Am J Forensic Med Pathol 1997; 18: II-I4.

I0.- Krous HF, Nadeau JM, Fukumoto RI, Blackbourne BD, Byard RW. Environmental hyperthermis infant and early childhood death. Circumstances, pathologis changes and manner of death. Am J Forensic Med Pathol 2001; 22:374-82.

II.- Whitehead FJ, Couper RTL, Moore L, Bourne AJ, Byard RW. Dehydration deaths in infants and young children. . Am J Forensic Med Pathol 1996; 17: 73-8. 